\title{
DINÂMICAS VEGETACIONAIS, CLIMÁTICAS E DO FOGO COM BASE EM PALINOLOGIA E ANÁLISE MULTIVARIADA NO QUATERNÁRIO TARDIO DO SUL DO BRASIL
}

\author{
SORAIA GIRARDI BAUERMANN \\ Laboratório de Palinologia, ULBRA, Av. Farroupilha, 8001, 92425-9000, Canoas, RS, Brazil.soraia.bauermann@ulbra.br \\ RENATO BACKES MACEDO \\ IG, UFRGS, Av. Bento Gonçalves, 9500, 91540-970, Porto Alegre, RS, Brazil. backesbiologia@yahoo.com.br
}

HERMANN BEHLING

Department of Palynology and Climate Dynamics, Albrecht-von-Haller-Institute for Plant Sciences, University of Göttingen, Untere Karspüle 2, 37073, Göttingen, Germany. hermann.behling@bio.uni-goettingen.de

VALÉRIO PILLAR

Departamento de Ecologia, UFRGS, Av. Bento Gonçalves, 9500, 91540-000, Porto Alegre, Brazil.vpillar@ufrgs.br

\section{PAULO CÉSAR PEREIRA DAS NEVES}

Universidade Luterana do Brasil, Av. Farroupilha, 8001, 92425-9000, Canoas, RS, Brazil.nevespc@yahoo.com.br

\begin{abstract}
RESUMO - A vegetação da região da campanha no Rio Grande do Sul é composta por formações campestres com raras e esparsas ocorrências de florestas de galeria. Uma importante questão discutida entre os botânicos é se no decorrer do Holoceno a paisagem, da região da campanha teve sempre predomínio de vegetação campestre como nos dias atuais ou se ocorreram formações florestais. Estudos palinológicos podem contribuir de maneira significativa para o entendimento desta questão. A análise preliminar da zona transicional entre as florestas e campos no Rio Grande do Sul, permite concluir que no Holoceno superior (3.000 anos A.P.) os elementos campestres dominaram o espectro polínico sobre os demais elementos pertencentes a outros tipos de formações. Significativo intercâmbio deu-se apenas dentro do mesmo hábito campestre, às vezes com prevalência de Poaceae e Baccharis (campos secos); outras vezes de Cyperaceae (campos úmidos).
\end{abstract}

Palavras-chave: Palinologia, Holoceno, Rio Grande do Sul, Brasil.

\begin{abstract}
SOUTHERN BRAZILIAN LATE QUATERNARY VEGETATION, FIRE AND CLIMATE DYNAMICS BASED ON PALYNOLOGICAL DATA AND MULTIVARIATE ANALYSIS. The vegetation of the campanha region of the Rio Grande do Sul state is composed by field formation, with rare and sparse gallery forests along some small rivers. Palynological research have been developed in others localities of the State, mainly in areas of the coastal plain and the plateau of Serra Geral. An important question, discussed among the botanicists is if during the Holocene the landscape of the campanha region was the same that it is now or if there were restrict forest formations. Palynological studies can significativaly contribute to the understanding of this question. A preliminary analysis in a transitional zone between forests and grasslands of the Rio Grande do Sul State allow to conclude that in the late Holocene (3.000 yr. B.P.) the grasslands absolutely dominated over other vegetational formations. Significant exchanges occurred only within the grasslands, sometimes with the prevalence of Poaceae and Baccharis type (dry fields), other times of Cyperaceae (wet/uliginous fields).
\end{abstract}

Key words: Palynology, Holocene, Rio Grande do Sul, Brazil.

\section{INTRODUÇÃO}

A vegetação do Estado do Rio Grande do Sul apresentase no Recente como um mosaico resultante das diferenças de relevo, solo, geologia e hidrografia. Esta vegetação, composta essencialmente de campos e florestas, encontrase em permanente competição no espaço regional e são condicionadas sob fortes influências ambientais, sobretudo as climáticas, que sofreram transformações ao longo do tempo e notavelmente durante o Quaternário. Esta paisagem já chamava atenção dos primeiros naturalistas que visitaram a região Sul do Brasil. No final do século XIX, Lindman (1906) concluiu que a vegetação campestre do Estado seria proveniente de um clima outrora mais seco. Posteriormente, Rambo (1956, 1961) e Klein (1975) complementaram as hipóteses de Lindman, sugerindo por evidências fitogeográficas que os Campos foram os primeiros tipos vegetais a se estabelecer nesta região do Brasil e, que a 
presença atual da grande diversidade de táxons florestais seria decorrente de um segundo momento quando houve mudanças no clima para condições mais úmidas.

Os estudos palinológicos são ferramentas ímpares para tais interpretações, principalmente, no Quaternário. Diversos estudos palinológicos já foram realizados na plataforma continental do Rio Grande do Sul (Lorscheitter, 1997, 2003; Bauermann \& Macedo, 2008), sobretudo, na Planície Costeira (Lorscheitter \& Dillenburg, 1998; Neves \& Lorscheitter, 1995; Macedo et al., 2007) e no Planalto (Behling et al., 2004; Behling et al., 2001; Leonhardt, 2007). Ainda sim, pouco se conhece sobre as dinâmicas vegetacionais, climáticas e do fogo em áreas interiorizadas como no "Planalto médio" e no Bioma Pampa, onde até o momento existem apenas dois estudos (Neves et al., 2001; Behling et al., 2005).

O trabalho aqui apresentado visa ampliar os conhecimentos sobre as principais mudanças paleovegetacionais ocorridas na região de São Martinho da Serra nos últimos milhares de anos através de estudos palinológicos e análise multivariada.

\section{ÁREA DE ESTUDO}

O município de São Martinho da Serra situa-se na região central do Rio Grande do Sul. Dista $260 \mathrm{~km}$ da capital do Estado, Porto Alegre e aproximadamente $40 \mathrm{~km}$ do município de Santa Maria (Figura 1). O ponto de coleta do perfil sedimentar (29 $27^{\prime} 21^{\prime \prime} \mathrm{S}$; 53 $\left.41^{\circ} 52^{\prime \prime} \mathrm{W}\right)$ encontra-se a 450 metros acima do nível relativo do mar.

A vegetação da região pertence à Floresta Estacional Decidual sendo uma zona transicional com os Campos sulrio-grandenses (Oliveira, 1991). Os campos apresentam-se bem diversificados sendo as espécies das famílias Poaceae, Fabaceae, Asteraceae e Rubiaceae as mais abundantes. A flora campestre está, também, representada por Apiaceae (Eryngium L., Hydrocotyle L.), Amaranthaceae (Alternanthera Forssk., Amaranthus L., Iresine P. Browne, Pfaffia Mart. ), Oxalidaceae (Oxalis L.), Alismataceae (Echinodorus Rich. ex Engelm., Sagittaria L.) e Smilacaceae (Smilax L.) entre outros. As espécies da Floresta Estacional Decidual pertencem, principalmente, as famílias Anacardiaceae (Lithrea Hook., Schinus L.), Aquifoliaceae (Ilex L.), Bignoniaceae (Jacaranda Juss., Arrabidaea DC.), Ebenaceae (Diospyros L.), Erythroxylaceae (Erythroxylum P. Browne), Euphorbiaceae (Euphorbia L., Sebastiania Bertol.), Flacourtiaceae (Casearia Jacq.), Lauraceae (Nectandra Rol. ex Rottb., Ocotea Aubl.), Melastomataceae (Miconia Ruíz \& Pav.), Meliaceae (Cabralea A. Jussieu, Cedrela P. Browne, Trichilia L.) e Ulmaceae (Celtis L., Trema Lour.).

O clima predominante no Estado do Rio Grande do Sul, conforme a classificação climática de Köppen, é do tipo climático " $C f a$ ", temperado com chuvas regularmente distribuídas durante o ano e com verões quentes. A temperatura média anual é de $17^{\circ} \mathrm{C}$ e a pluviosidade média anual oscila entre 1.250 e $2.000 \mathrm{~mm}$ (Nimer, 1989).

Os os jesuítas foram os primeiros colonizadores europeus a se estabelecer na região quando fundaram a redução de São Miguel (Lessa, 2002). Junto trouxeram os primeiros bovinos que se procriaram rapidamente formando as vacarias. A partir do século XIX, houve a chegada dos imigrantes portugueses os quais sobreviviam basicamente da extração da erva-mate. Posteriormente, com a chegada dos imigrantes alemães atraídos pelos terrenos pouco acidentados, de solo fértil, terra barata e fácil de trabalhar e, principalmente pela inexistência de grandes florestas, iniciou-se o desenvolvimento da cidade de São Martinho da Serra.

\section{MATERIAIS E MÉTODOS}

O perfil sedimentar foi extraído na porção central de uma pequena bacia sedimentar com auxílio do equipamento coletor "Russian" atingindo uma profundidade total de 100 centímetros. Três subamostras foram retiradas do perfil sedimentar para datações por "Accelerator Mass Spectrometry" (AMS) e as idades obtidas foram calibradas com base em CALPAL (Weninger et al., 2004). Em laboratório foram retiradas 50 unidades amostrais, em intervalos regulares ao longo do perfil, com volume de $01 \mathrm{~cm}^{3}$ cada. As subamostras foram processadas quimicamente seguindo a técnica de acetólise, conforme Faegri \& Iversen (1989). Após a preparação do material, foram confeccionadas lâminas permanentes pelo método de gelatina glicerinada, SalgadoLabouriau (1973).

Para determinar os cálculos de concentração polínica (grãos $/ \mathrm{cm}^{3}$ ) foram adicionados em cada unidade amostral dois tabletes de Lycopodium clavatum, conhecendo-se número exato de esporos/tablete, segundo Stockmarr (1971). A identificação taxonômica dos palinomorfos foi realizada com auxílio de bibliografias especializadas, mais especificamente, Hooghiemstra (1984); Roubik \& Moreno (1991); Colinvaux et al., (1999), Neves \& Bauermann (2003, 2004), Neves \& Cancelli (2006), além de consultas a palinoteca contendo grãos de pólen e esporos típicos da região. Em todas as unidades amostrais foram contados no mínimo 300 grãos de pólen, enquanto os demais palinomorfos foram contados a parte $\mathrm{e}$ todos foram agrupados conforme suas afinidades ecológicas, hábito e/ou hábitat. A soma polínica incluiu elementos herbáceos, arbóreo-arbustivos e grãos de pólen indeterminados. Os táxons de macrófitos aquáticos, esporos de pteridófitos e briófitos, colônias de algas, bem como as partículas carbonizadas (entre 5-150 $\mu \mathrm{m}$ ), também foram contados e expressos em porcentagem sob o total do somatório polínico. Para ilustrar e analisar os dados polínicos foram utilizados os softwares TILIA, TGVIEW e CONISS Grimm (1987). Análise multivariada de ordenação por Análise de Coordenadas Principais (PCoA) foi aplicada com vistas a obter uma síntese da trajetória de mudanças na composição polínica da vegetação (Orlóci et al., 2002). PCoA foi baseada em distâncias euclidianas entre pares de unidades amostrais (Podani, 2000; Legendre \& Legendre, 1998). As distâncias foram calculadas a partir das porcentagens de composição polínica transformadas por raiz quadrada, para reduzir distorções causadas pelo peso excessivo de táxons 


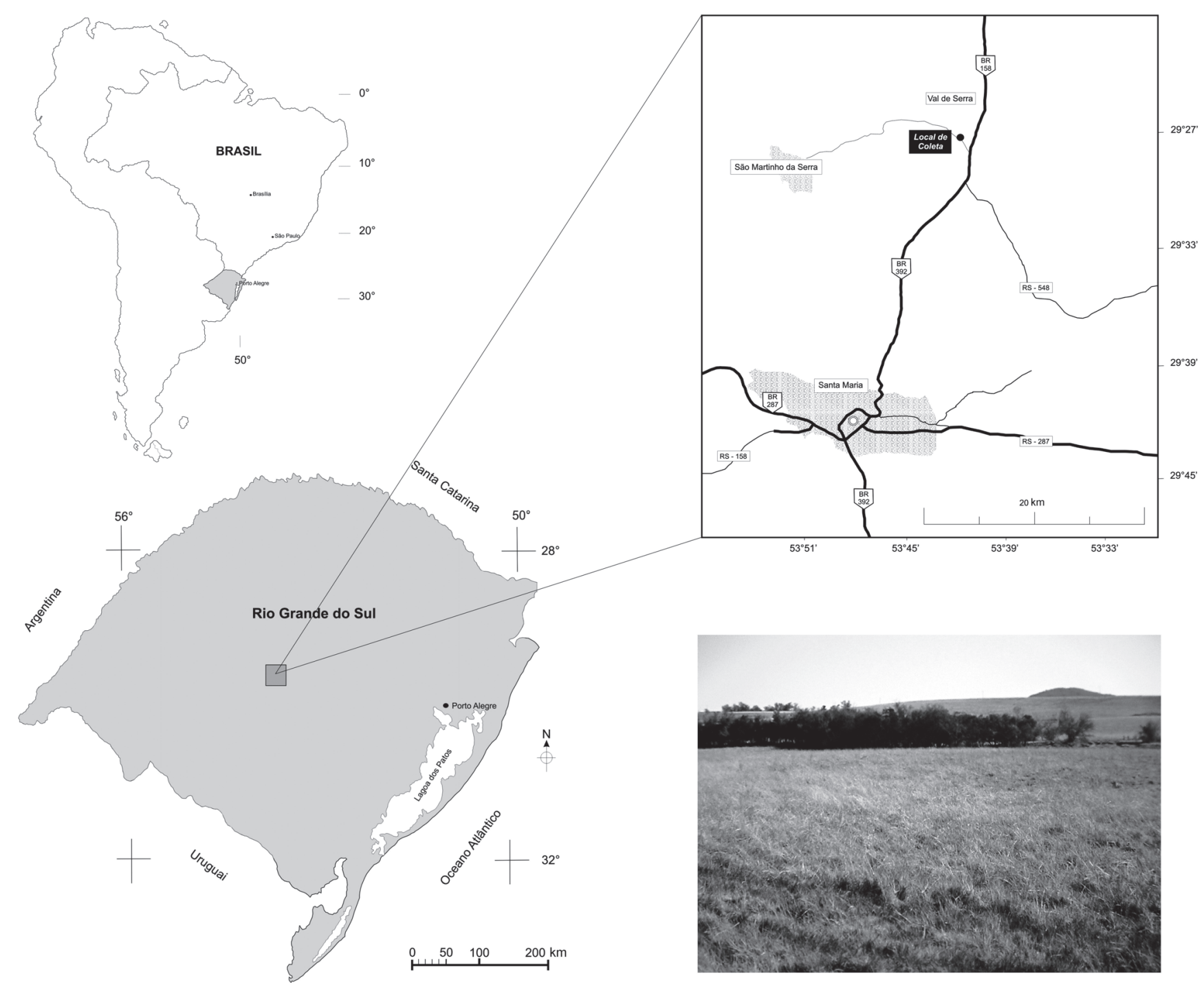

Figura 1. Mapa de localização da área estudada com indicação do local de perfuração e fotografia da região.

Figure 1. Location map of study area showing the sampled site and photograph from the region.

dominantes. Na análise de ordenação foram ignorados táxons indeterminados, táxons que produzem esporos, táxons aquáticos e Cyperaceae, com vistas a enfatizar na análise a variação regional da vegetação. A significância dos eixos de ordenação foi avaliada por auto-reamostragem (Pillar, 1999). As taxas de mudança na composição polínica foram calculadas dividindo-se a distância euclidiana pelo respectivo intervalo de tempo entre unidades amostrais adjacentes no perfil sedimentar. Com os mesmos dados foi calculada a riqueza e a diversidade (índice de Shannon) de táxons polínicos. As análises foram realizadas com o software MULTIV (Pillar, 2008).

\section{RESULTADOS}

Cronologia. As datações radiocarbônicas realizadas pela técnica de (AMS) indicaram idades holocênicas para o depósito sedimentar, acusando aproximadamente $3.231 \pm 42$ anos A.P. na base do testemunho de sondagem. A segunda datação, realizada a $79 \mathrm{~cm}$ de profundidade, apontou para $1.574 \pm 42$ anos A.P. Uma terceira amostra extraída a $21 \mathrm{~cm}$ registrou idade moderna.

Tabela 1. Profundidade $(\mathrm{cm})$ e sedimentos dos níveis amostrados neste estudo.

Table 1. Depth $(\mathrm{cm})$ and sediments of the sampled levels in this study.

\begin{tabular}{c|l}
\hline $\mathbf{c m}$ & \multicolumn{1}{c}{ Descrição } \\
\hline $\mathbf{0}-\mathbf{3 0}$ & $\begin{array}{l}\text { Turfa fibrosa com presença de raízes; } \\
\text { material inconsolidado de cor castanha. }\end{array}$ \\
\hline $\mathbf{3 0 - \mathbf { 8 0 }}$ & $\begin{array}{l}\text { Turfa hêmica com matéria orgânica } \\
\text { totalmente decomposta, compactada, } \\
\text { preta. }\end{array}$ \\
\hline $\mathbf{8 0 - 1 0 0}$ & $\begin{array}{l}\text { Argila de coloração castanho-acizentado } \\
\text { com presença de areia fina. }\end{array}$ \\
\hline
\end{tabular}



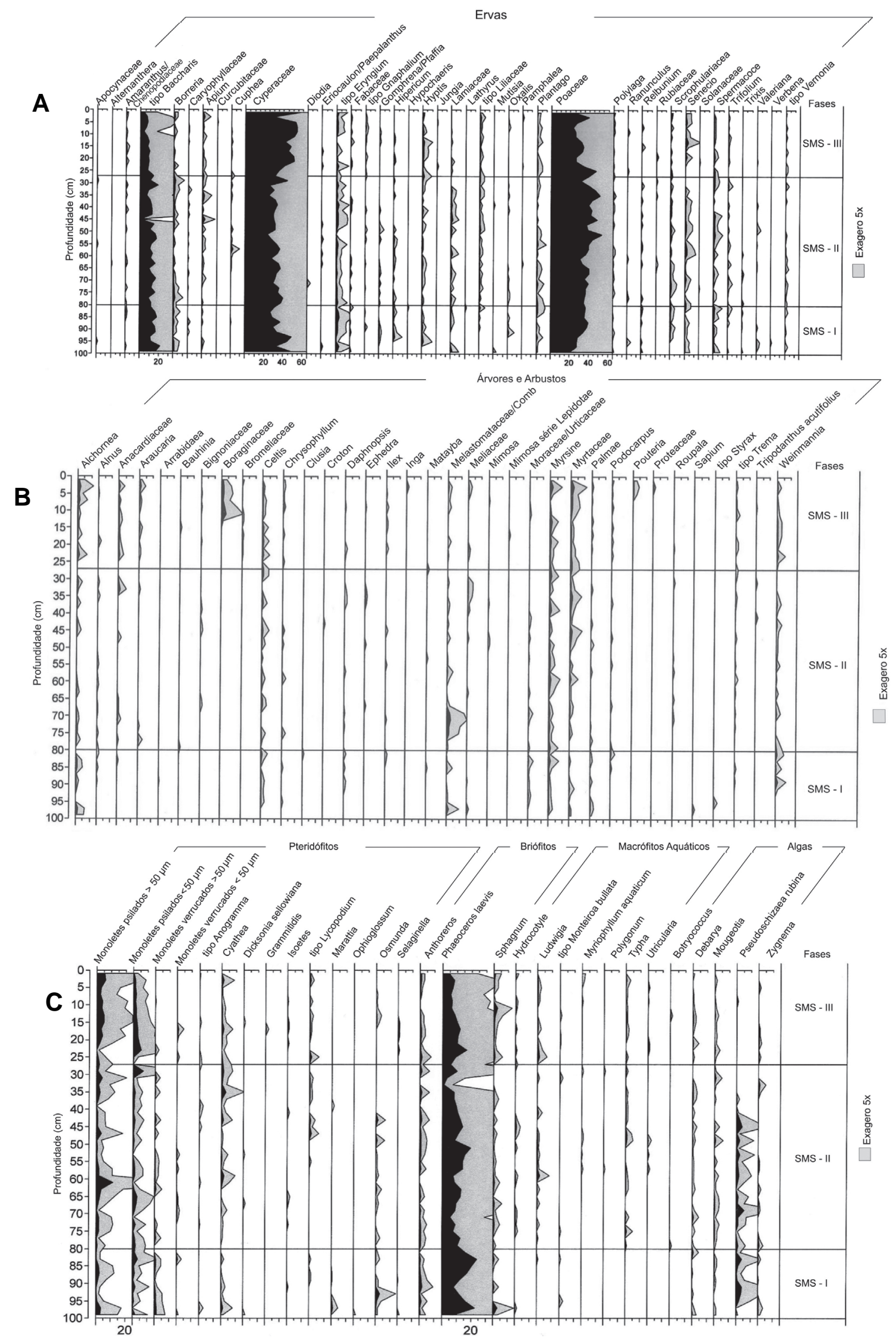

Figuras 2. Diagrama polínico de porcentagem mostrando a ecologia dos grupos: A, ervas; B, árvores e arbustos; C, pteridófitos, briófitos, áquaticos e algas.

Figures 2. Pollen percentage diagrams showing the ecology of groups: A, herbs; B, trees and shrubs; C, pteridophytes, briophytes, aquatic plants and algae. 
Dados sedimentológicos. As características sedimentares e de coloração das amostras coletadas mostraram poucas variações ao longo do testemunho de sondagem (Tabela 1). Dados Polínicos. Os diagramas polínicos de São Martinho da Serra (Figuras 2A, 2B, 2C) mostraram a porcentagem dos 105 palinomorfos identificados os quais foram categorizados conforme suas afinidades ecológicas. As análises de agrupamentos (CONISS) permitiram averiguar as principais mudanças paleoflorísticas ocorridas nos conjuntos polínicos sendo possível estabelecer três fases ecológicas distintas, SMS - I; SMS - II; SMS - III, (Figura 3).

A Fase SMS - I, $(100-80 \mathrm{~cm}, 3.231 \pm 42-1.574 \pm 42$ anos A.P., 10 unidades amostrais) é marcada pela abundância de grãos de pólen de plantas herbáceas (89-97\%), sendo dominados por Cyperaceae (27-49\%), Poaceae (19-39\%), tipo Baccharis (11-21\%), tipo Eryngium, Hyptis, e Plantago, além de muitos outros táxons herbáceos com menores índices percentuais. O grupo de árvores e arbustos, consistindo principalmente de Alchornea, Celtis, Melastomataceae/ Combretaceae, Moraceae/Urticaceae, Myrsine, Myrtaceae, Palmae, Weinmannia e alguns outros táxons com baixas porcentagens, tiveram soma polínica apenas entre $0,5-5 \%$. Porcentagem de grãos de pólen de plantas aquáticas como Hydrocotyle, Ludwigia e tipo Monteiroa bullata perfazem menos de 1\%. Esporos de pteridófitos foram relativamente escassos em todas as três fases (2,5-13\%). Esporos de Dicksonia sellowiana foram raramente encontrados e tiveram percentuais menores do que $0,5 \%$. Os briófitos foram melhores representados por esporos de Phaeoceros laevis (23\%), enquanto Anthoceros e Sphagnum foram raros. A concentração de partículas carbonizadas foram relativamente altas, principalmente em níveis basais.

Na Fase SMS - II, $(80-27 \mathrm{~cm}, 1.574 \pm 56$ A.P. -468 anos cal. A.P., 27 unidades amostrais) os grãos de pólen dos táxons herbáceos apresentaram valores percentuais semelhantes a fase anterior (73-96\%), entretanto, este intervalo foi caracterizado pela mudança na composição florística dos elementos de campo, com aparecimento de táxons como Alternanthera, Diodia, Pamphalea e Rubiaceae, Solanaceae, além do aumento na freqüência relativa de Poaceae (máx. $54 \%$ ) e o decréscimo de grãos de pólen de Cyperaceae (mín. 22\%). O percentual de tipo Baccharis permaneceu constante com valor de $16 \%$. Os elementos arbóreos-arbustivos mantiveram-se com valores percentuais muito baixos (inferior a $6 \%$ ) sendo mais bem representados pela ocorrência de elementos tais como Melastomataceae/Combretaceae (máx. 3\%), Myrsine e Myrtaceae (ambos com máx. 1,5\%). Também surge no registro táxons como Araucaria angustifolia, Bauhinia, Bignoniaceae, Croton, Mimosa, Roupala, além de elementos associados como Ephedra e Tripodanthus

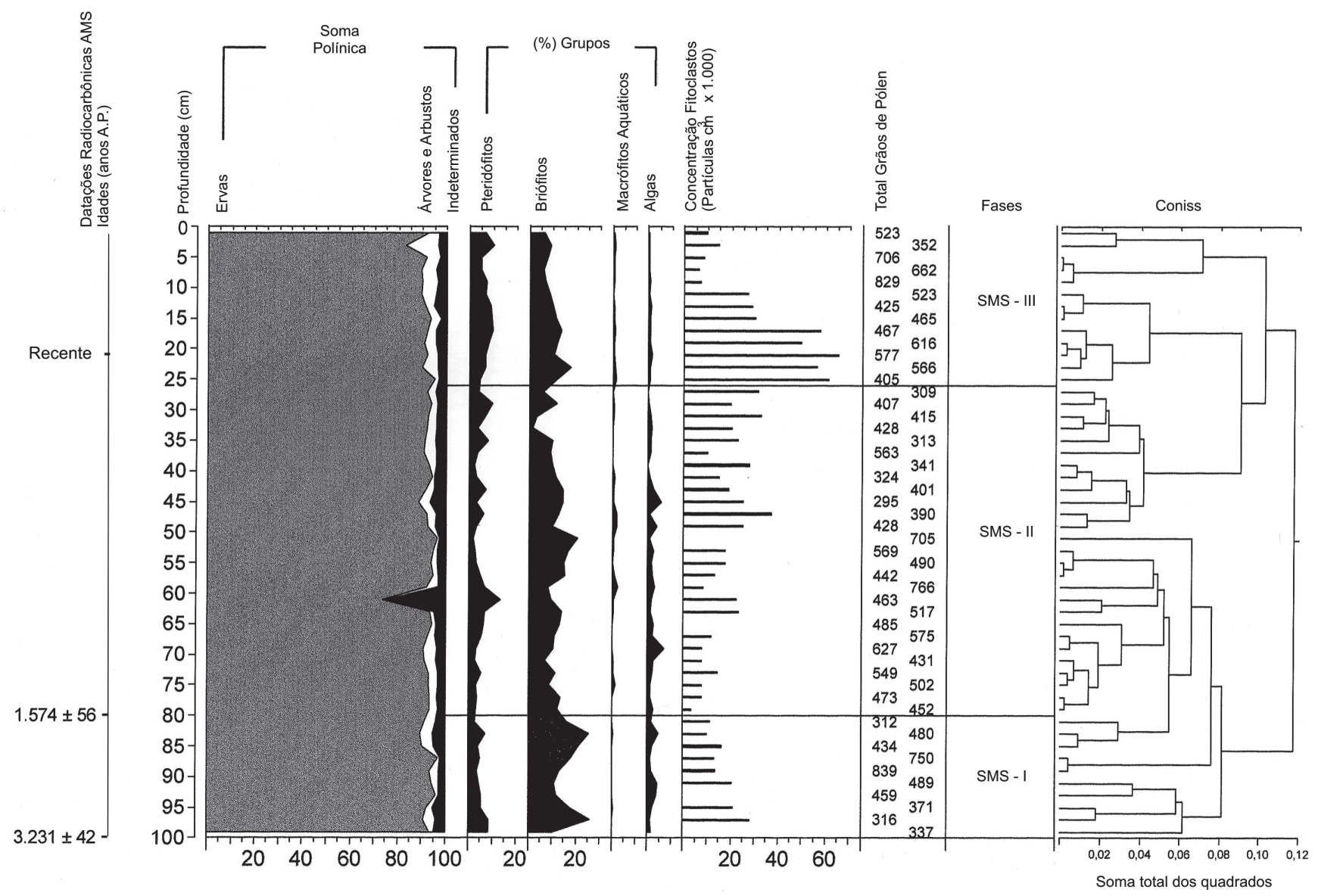

Figura 3. Diagrama da soma polínica de percentagem, níveis datados, ecologia dos grupos, zonas polínicas e dendrograma. Figure 3. Diagram of the pollen sum, dating levels, groups ecology, pollen zones and dendrogram. 

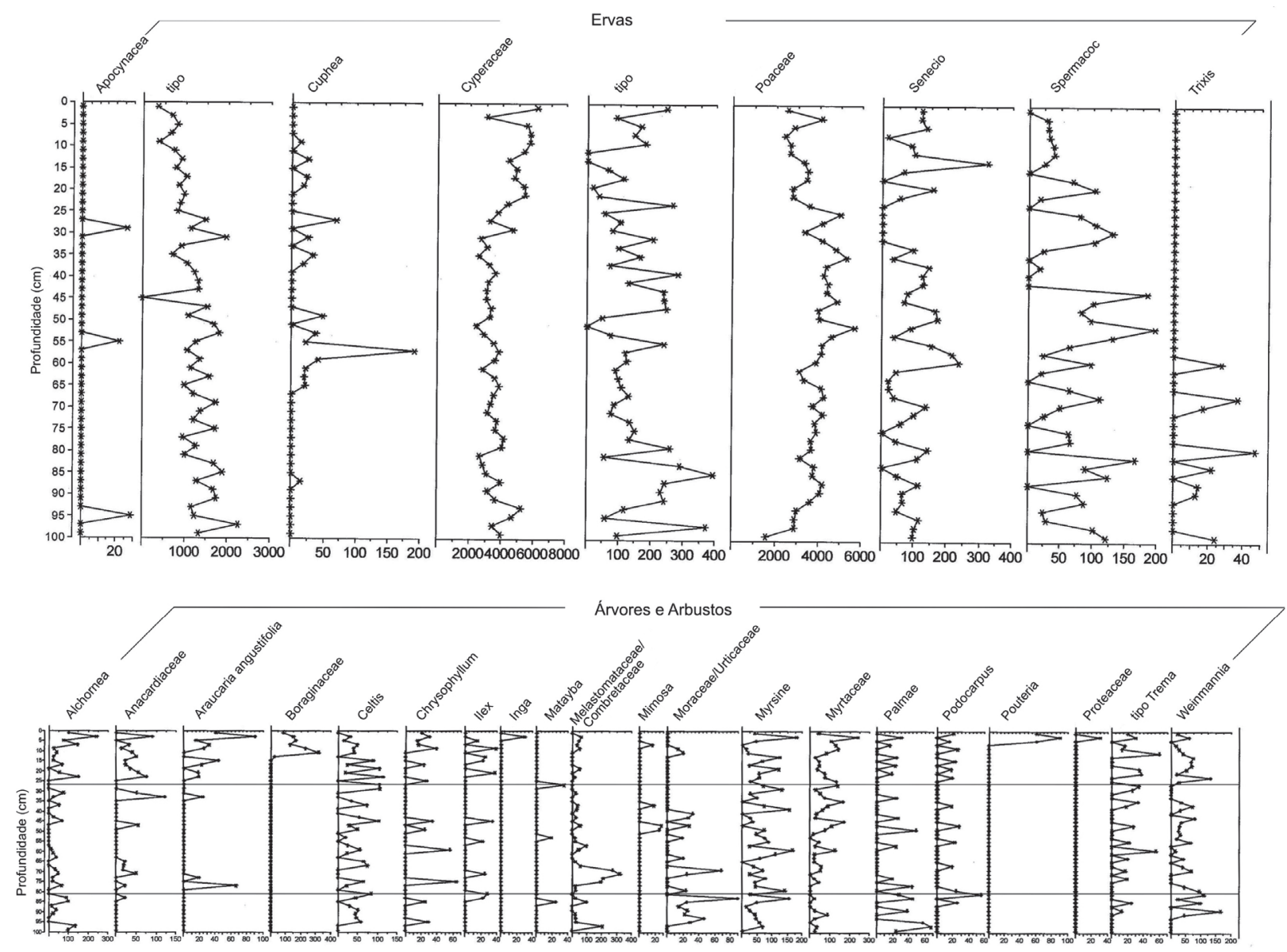

Figura 4. Diagrama de concentração dos principais esporomorfos registrados em São Martinho da Serra: ervas (acima), árvores e arbustos (abaixo).

Figure 4. Concentration diagram of main sporomorphs registred in São Martinho da Serra: herbs (top), trees and shrubs (bottom).

acutifolius. Esporos de Phaeoceros laevis, assim como na fase anterior, apresentaram taxas percentuais elevadas em torno de $20 \%$, mas tiveram oscilações ao longo deste período declinando de forma acentuada ao término deste. A concentração de partículas carbonizadas aumentou e apresentou percentuais mais elevados no terço final deste intervalo.

A Fase SMS - III, (27-0 cm, 468 anos cal. A.P. - Recente, 13 unidades amostrais) confirmaram o observado para as duas fases anteriores no que diz respeito à boa representação das formas campestres (82-95\%). Os percentuais de tipo Baccharis (3-10\%) e Poaceae (23-46\%) decrescem, enquanto Cyperaceae aumenta atingindo 58\% (Figura 2A, 4A). Aparecem no registro Curcubitaceae e Jungia e, desaparecem Apocynaceae, Alternanthera, Diodia, Mutisia e Trixis. Alguns elementos representativos de árvores e arbustos como Alchornea, Anacardiaceae, Boraginaceae, Celtis, Chrysophyllum, Melastomataceae, Meliaceae, Myrsine, Myrtaceae, Podocarpus e Weinmannia tiveram aumento em suas freqüências relativas contribuindo para o aumento percentual na soma deste grupo (4-14\%), (Figura 2B, 4B). No inicio da fase SMS SMS - III observou-se a maior concentração de partículas carbonizadas.

Análise multivariada. A análise multivariada de ordenação
(Figura 5) mostra de forma sintética a trajetória de mudança da composição polínica de 3427 anos cal. A.P. até o presente. Até cerca de 1427 anos cal. A.P. (SMS - I) a composição foi caracterizada por maiores abundâncias relativas de Eryngium, Melastomataceae, Borreria, Sapium, Alchornea e Baccharis. Esse período foi seguido por uma tendência a aumento de Poaceae na fase SMS - II. Após 429 anos cal. A.P., na fase SMS - III, a trajetória mostrou uma mudança marcada em direção a maiores abundâncias relativas de Boraginaceae, Araucaria, Myrtaceae, Alchornea e Bromeliaceae. A velocidade de mudança da composição polínica (Figura 6) foi mais lenta até 1427 anos cal. A.P. (fase SMA-I) e mais rápida após. A diversidade da composição polínica ao longo do período mostrou pequena variação, com leve tendência de aumento no período recente (Figura 7).

\section{DISCUSSÃO}

Os resultados das análises polínicas do perfil sedimentar de São Martinho da Serra mostraram que as terras baixas do oeste do Rio Grande do Sul foram naturalmente coberta por Campos desde o "Holoceno médio" até o Recente.

Os dados palinológicos na base do sítio deposicional (3.231 \pm 2 anos A.P.) atestaram que o ambiente paludial de 


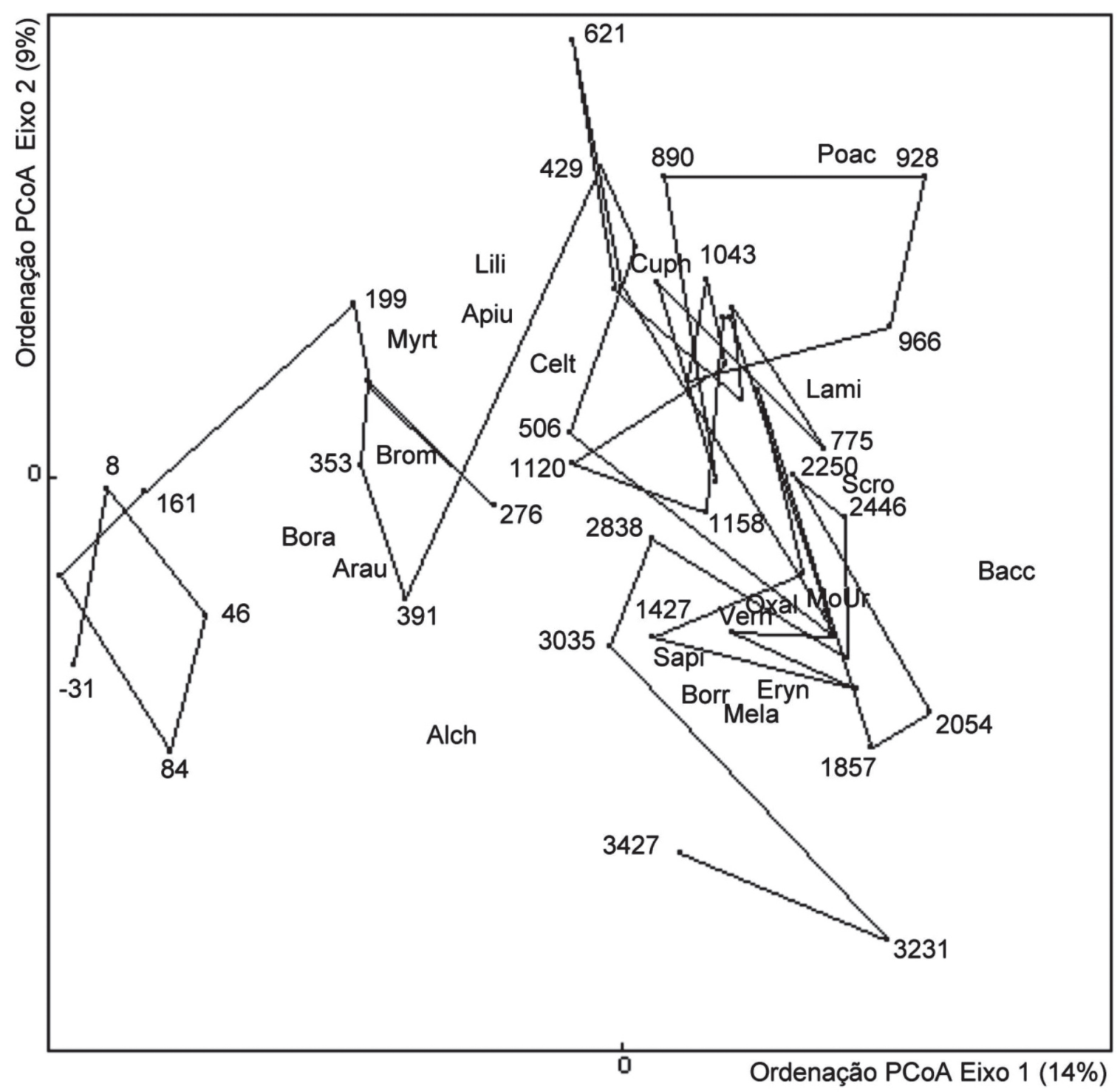

Figura 5. Diagrama de ordenação com base na composição polínica do perfil sedimentar de São Martinho. A trajetória conecta subamostras desde 3427 anos calibrados A.P. até o presente. Método de ordenação por coordenadas principais, a partir de distâncias euclidianas entre subamostras, calculadas com as abundâncias porcentuais de 75 táxons transformadas por raiz quadrada. Abreviaturas: Alch, Alchornea; Apiu, Apium; Arau, Araucaria angustifolia; Bacc, Baccharis type; Bora, Boraginaceae; Brom, Bromeliaceae; Borr, Borreria; Celt, Celtis; Cuph, Cuphea; Eryn, Eryngium type; Lami, Lamiaceae; Lili, Liliaceae type; Mela, Melastomataceae; MoUR, Moraceae/Urticaceae; Myrt, Myrtaceae; Oxal, Oxalis; Poac, Poaceae; Sapi, Sapium; Scro, Scrophullariaceae; Vern, Vernonia type.

Figure 5. Ordination diagrams based on pollen taxa described from sediment core of São Martinho, RS. The trajectory connects subsamples from 3427 years AP calibrated to the present. Ordination method is principal coordinates analysis based on euclidian distances between sampling units, calculated with percentage abundance of 75 taxa transformed by square root. Abbreviations as above.

São Martinho da Serra provavelmente evoluiu a partir de um pequeno lago raso que já apresentava sinais de colmatação devido a presença e abundância no registro de ciperáceas, macrófitos aquáticos e algas (Figuras $2 \mathrm{C}$ e 3 ); enquanto a ocorrência de Phaeoceros laevis indica calor. Os raros grãos de pólen pertencentes a formações florestais, documentaram uma baixa diversidade arbóreo/arbustiva, resultados também encontrados nas regiões de São Gabriel (Neves et al., 2001) e São Francisco de Assis (Behling et.al., 2005). Estas incipientes formações florestais estavam constituídas principalmente por Alchornea, Anacardiaceae, Celtis, Ilex, Melastomataceae/Combretaceae, Moraceae/Urticaceae, Myrsine, Myrtaceae, Palmae, Podocarpus e Weinmannia. A presença no espectro polínico de Podocarpus, Ilex, Myrsine e Myrtaceae aliada ao registro de Dicksonia sellowiana sugere fitofisionomia semelhante a Floresta Ombrófila Mista que tem, na atualidade, estes táxons como seus elementos característicos.

Em torno de $1.574 \pm 42$ anos A.P., houve uma troca significativa na composição florística dentre os elementos campestres com o surgimento de táxons como Apocynaceae, Cuphea, Diodia, Mutisia e aumento de Poaceae, Senecio e Spermacoce. A vegetação arbórea/arbustiva também diversificou-se com o surgimento de elementos como Araucaria angustifolia, Mimosa, Roupala e Tripodhantus acutifolius, sinalizando um aumento de umidade para esta 


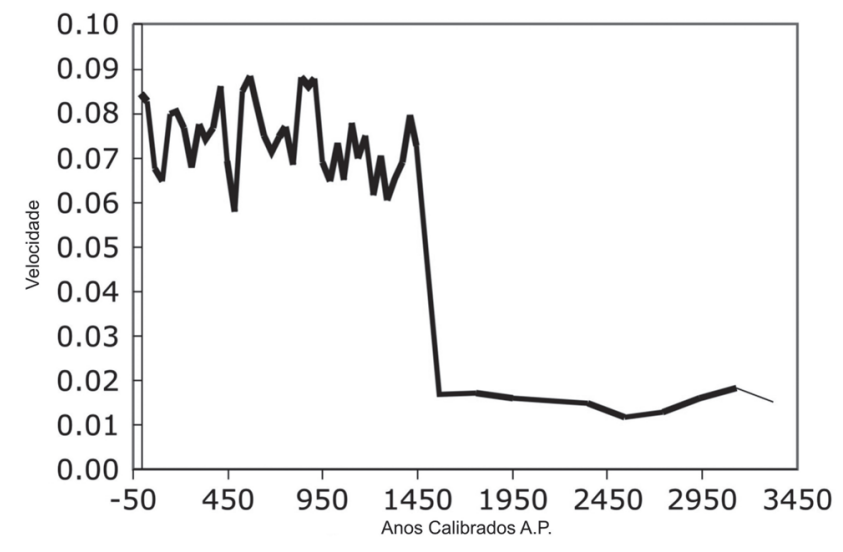

Figura 6. Velocidade de mudança na composição polínica do perfil com base em distâncias euclidianas entre subamostras, calculadas com as porcentagens de 75 táxons previamente transformadas por raiz quadrada.

Figure 6. Change speed in the pollen composition based on subsample euclidian distances calculated with the percentages of 75 taxa previously transformed by square root.

idade. Diversificação nas formações florestais por volta de $1.574 \pm 42$ anos A.P. também foram encontrados em outros sítios do Rio Grande do Sul (Bauermann, 2003; Behling et al., 2005; Macedo et al., 2007). O aumento de porcentagem na concentração de fitoclastos (Figura 3) nesta fase mostra que o fogo também pode estar associado à mudança da vegetação em direção a maior ocorrência de Poaceae nesse período.

Na transição da fase SMS - II para a SMS - III evidenciouse um importante aumento nas taxas de concentração das partículas carbonizadas. Provavelmente o fogo no início desse período tenha sido de origem antrópica, embora não se descarte influência climática favorecendo o aumento de produção de biomassa, que por sua vez acentua a flamabilidade da vegetação. Evidências arqueológicas corroboram as interpretações correlacionando o fogo a origem antrópica por grupos caçadores e coletores na região dos campos (Schmitz, 2005).

A última fase, situada integralmente no Recente, continua com hegemonia das formações campestres sobre as florestais. A diversidade da vegetação herbácea declinou suportado pelo desaparecimento de registros de Apocynaceae, Alternanthera, Diodia, Hypochaeris, Lathyrus, Mutisia, Trixis e Verbena, sinal, provavelmente, da atividade humana devido a criação de gado. As formações florestais também apresentaram trocas na composição florística com o desaparecimento de Clusia e Croton; e o surgimento de Inga, Pouteria, Proteaceae, Boraginaceae, Bromeliaceae (epífito) enquanto Alchornea, Anacardiaceae, Araucaria angustifolia, Chrysophyllum, Myrsine, Trema, Myrtaceae e Weinmannia aumentaram suas ocorrências (Figura 4B). A presença dos novos táxons aponta para expansão de elementos da Floresta Estacional Decidual, atualmente presente nesta região.

A presença de Alnus, ao longo do furo de sondagem, atestou a ocorrência de correntes de vento oeste assolando o RS e trazendo elementos exóticos a flora local, uma vez que

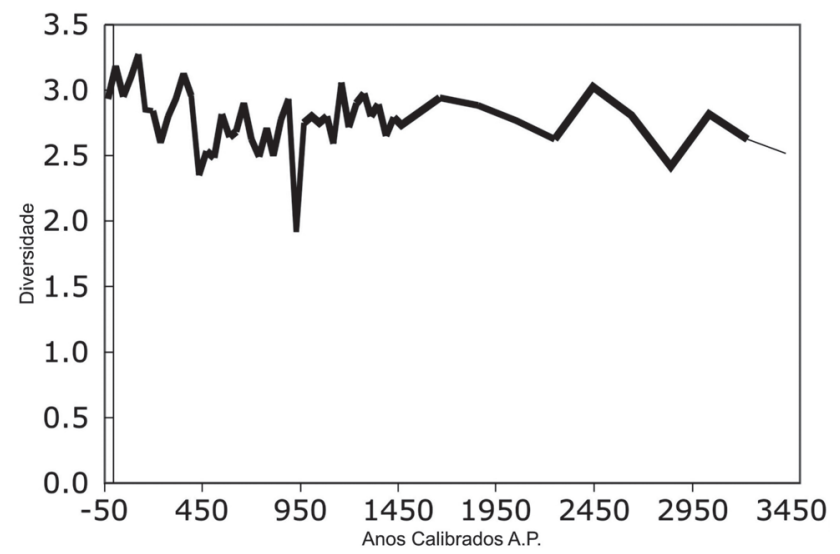

Figura 7. Diversidade (Shannon) da composição polínica do perfil sedimentar.

Figure 7. Diversity (Shannon) of the pollen composition from the sedimentary profile.

este gênero tem sua ocorrência no sudoeste da América do Sul.

Os resultados obtidos em São Martinho da Serra são semelhantes àqueles observados nos municípios de São Gabriel (Neves et al., 2001) e São Francisco de Assis (Behling et al., 2005), que também pertencem ao domínio dos campos sul-rio-grandenses).

A ausência de registro de grãos de pólen relacionados a agricultura é condizente com os dados arqueológicos uma vez que as regiões habitadas pelos paleoindígenas horticultores são distantes do local estudado (cerca de127 $\mathrm{km}$ ). A chegada do colonizador, na região de São Martinho da Serra, também não deixou marcas evidentes no registro polínico uma vez que os mesmos sobreviviam do extrativismo da erva-mate e pecuária.

O leve decréscimo de táxons florestais, no topo do perfil sedimentar, deve estar relacionado com o processo de desmatamento que vem ocorrendo indiscriminadamente nos dias atuais.

\section{CONCLUSÕES}

Os níveis amostrados no testemunho de sondagem permitiram identificar 105 diferentes tipos de palinomorfos que evidenciaram, por sua distribuição e freqüência, o amplo predomínio dos Campos desde o "Holoceno médio" até os dias atuais.

As análises polínicas da área em estudo mostraram que as formações florestais existentes deveriam estar situadas ao longo dos rios formando as matas de galeria, e nas baixadas úmidas constituindo pequenas áreas florestadas. O aumento da umidade, ocorrido sobretudo após $1.574 \pm 42$ anos A.P., é indicado pela expansão e diversificação das formações florestais. O surgimento e incremento, em tempos modernos, de táxons como Inga, Melastomataceae/Combretaceae, Pouteria e Myrtaceae demonstram a invasão de elementos da mata latifoliada sobre a floresta já estabelecida.

Várias hipóteses existem de rotas migratórias para os contingentes vegetacionais do Estado. Segundo Rambo 
(1961), a leste, houve migração da flora tropical atlântica que penetrou pela planície costeira, contornou o Planalto e rumou para oeste. As Matas das Bacias Paraná/Uruguai, a oeste, migraram para leste através das bordas do Planalto. Evidências destas duas rotas migratórias foram fornecidas, respectivamente, pelos registros de grãos de pólen de Alchornea, Myrsine, Moraceae/Urticaceae característicos da Mata Atlântica e por Chrysophyllum, Matayba, Melastomataceae/Combretaceae indicativos da presença das Matas da bacia Paraná/Uruguai. Considerando a dispersão polínica de Araucaria angustifolia em torno de $600 \mathrm{~m}$ (Caccavari 2003), o registro desde 3.271 anos A.P. de grãos de pólen desta espécie, comprova a ocorrência natural desta espécie nas baixadas das colinas e nas matas de galeria.

Os dados paleovegetacionais indicaram a vigência da vegetação de Campos sob condições de calor e umidade do "Holoceno superior" perdurando mesmo sob a intensificação destas, fato ocorrido após 1574 anos A.P. O registro das partículas carbonizadas atingiu seu pico de representatividade por volta de $468 \mathrm{cal}$. anos A.P. demonstrando, provavelmente, que seu acréscimo está relacionado ao processo de colonização humana.

Os dados paleopolínicos, obtidos neste estudo, acerca da composição florística dos biomas originais, fornecem resultados ímpares para a recuperação de áreas degradadas e manejo dos remanescentes florestais. A compreensão da dinâmica paleovegetacional pode servir de base para a construção de modelos regionais de implantação e aceleração do processo de sucessão vegetacional, bem como constituir banco de dados na busca de essências nativas para projetos de reflorestamento.

\section{REFERÊNCIAS}

Bauermann, S.G. 2003. Análises palinológicas e evolução paleovegetacional e paleoambiental das turfeiras de Barrocadas e Águas Claras, Planície Costeira do Rio Grande do Sul, Brasil. Programa de Pós-graduação em Geociências, Universidade Federal do Rio Grande do Sul, Tese de Doutorado, 137 p.

Bauermann, S.G. \& Macedo, R.B. 2007. Dinâmicas paleovegetacionais, paleoclimáticas e evidências antrópicas baseado em dados polínicos no Rio Grande do Sul. In: WORKSHOP QUATERNÁRIO DO RIO GRANDE DO SUL, 2007, Resumos, Canoas, ULBRA, p.35-36.

Behling, H.; Bauermann, S.G. \& Neves, P.C.P. das. 2001. Holocene environmental changes in the São Francisco de Paula region, southern Brazil. Jounal of South American Earth Science, 14:631-639.

Behling, H.; Pillar, V. \& Bauermann, S.G. 2004. Late Quaternary Araucaria forest, grassland (campos), fire and climate dynamics, inferred from a high-resolution pollen record of Cambará do Sul in southern Brazil. Palaeogeograph, Palaeoclimatology, Palaeoecology, 203:277-297.

Behling, H.; Pillar, V.D.; Bauermann, S.G. 2005. Late Quaternary grassland (Campos), gallery forest, fire and climate dynamics studied by pollen, charcoal and multivariate analysis of the São Francisco de Assis core in western Rio Grande do Sul (southern Brazil). Review of Paleobotany and Palynology, 133:235-248.

Caccavari, M.A. 2003. Dispersión del pollen en Araucaria angustifolia (Bert.) O. Kuntze. Revista do Museo Argentino de Ciencias Naturales, 5(2):135-138.

Colinvaux, P.; De Oliveira, P.E. \& Patinõ, J.E.M. 1999. Amazon pollen manual and atlas. Harwood Academic Publisher, Amsterdam, $330 \mathrm{p}$.

Faegri, K. \& Iversen, J. 1989. Textbook of Pollen Analysis. John Wiley \& Sons, New York, 328 p.

Grimm, E.C. 1987. CONISS: A Fortran 77 program for stratigraphically constrained cluster analysis by the method of the incremental sum of squares. Pergamon Journal, 13:13-35.

Hooghiemstra, H. 1984. Vegetacional and climatic history of the High Plain of Bogotá, Colombia: a continuous record of the last 3,5 million years. Dissertationes Botanicae, 79:1-368.

Klein, R.M. 1975. Southern Brazilian phytogeographic features and the probable influence of upper Quaternary climatic changes in the floristic distribution. Boletim Paranaense de Geociências, 33:67-88.

Leonhardt, A. 2007. Mudanças Vegetacionais e Climáticas no Planalto Leste do Rio Grande do Sul, Brasil, durante os últimos 25000 anos. Programa de Pós-Graduação em Biociências, Universidade Federal do Rio Grande do Sul, Dissertação de Mestrado, $124 \mathrm{p}$.

Legendre, L. \& Legendre, P. 1998. Numerical Ecology. 2nd ed. New York, Elsevier, 853 p.

Lessa, L.C.B. 2002. Rio Grande do Sul, prazer em conhecê-lo: como surgiu o Rio Grande. $4^{\mathrm{a}}$ ed. Porto Alegre, 191 p.

Lindman, C.A.M. 1906. A vegetação no Rio Grande do Sul. EDUSP/ Itatiaia, São Paulo/ Belo Horizonte, Brasil, 377 p.

Lorscheitter, M.L. 1997. Paleoambientes do Sul do Brasil no Quaternário através da palinologia: Revisão dos resultados obtidos. Revista Universidade de Guarulhos, Geociências II ( $\mathrm{n}^{\circ}$ especial): 197-199.

Lorscheitter, M.L. 2003. Contribution to the Holocene history of Atlantic rain forest in the Rio Grande do Sul state, southern Brazil. Rev. Mus. Argentino Cienc. Nat, 5(2): 261-271.

Lorscheitter, M.L. \& Dillenburg, S.R. 1998. Holocene palaeoenvironmental of the northern coastal plain of Rio Grande do Sul, Brazil, reconstruteced from palynology of Tramandaí lagoon sediments. Quaternary of South America and Antartic Peninsula, 11:73-98.

Macedo, R.B.; Cancelli, R.R.; Bauermann, S.G.; Neves, P.C.P. das \& Bordignon, S.A.de L. 2007. Palinologia de níveis do Holoceno da Planície Costeira do Rio Grande do Sul (localidade de Passinhos), Brasil. Revista Gaea Unisinos 3(2):68-74.

Neves P.C.P. das. \& Bauermann, S.G. 2003. Catálogo palinológico de coberturas quaternárias do Estado do Rio Grande do Sul (Guaíba e Capão do Leão), Brasil. Descrições Taxonômicas Parte - I: fungos, algas, palinomorfos outros e fragmentos de invertebrados. Pesquisas Série Botânica, 53: 121-149.

Neves P.C.P. das. \& Bauermann, S.G. 2004. Catálogo palinológico de coberturas quaternárias do Estado do Rio Grande do Sul (Guaíba e Capão do Leão), Brasil. Descrições Taxonômicas Parte - II: Bryophyta e Pteridophyta. Pesquisas Série Botânica, 55: 227-251.

Neves, P.C.P. das; Bachi, F.A.; Rossoni, M.G.; Bauermann, S.G.; Bordignon, S.A. de L., Kroeff, V.N.; Groff, A. \& Souza, A. L. F. de. 2001. Palinologia de um depósito paludoso na região do banhado do Jacaré, campanha do estado do Rio Grande do Sul, Brasil. In: CONGRESSO DAASSOCIAÇÃO BRASILEIRA DE ESTUDOS DO QUATERNÁRIO, 8, 2001. Resumos Expandidos, Mariluz-Imbé: p. 365-367.

Neves, P. C. P. das. \& Cancelli, R. R. 2006. Catálogo palinológico 
em sedimentos do final do Neógeno no Estado do Rio Grande do Sul (Guaíba e Capão do Leão), Brasil. Taxonomia - Parte IV: Magnoliophyta I (Magnoliopsida). Revista Gaea Unisinos, 2(2):75-89.

Neves, P.C.P. das \& Lorscheitter, M.L. 1995. Upper Quaternary palaeoenvironments in the Northern Coastal Plain of Rio Grande do Sul, Brazil. Quaternary of South America and Antarctic Peninsula, 9:39-67.

Nimer, E. 1989. Climatologia do Brasil. Rio de Janeiro, Fundação Instituto Brasileiro de Geografia e Estatística, 421 p.

Oliveira, M.L.A.A. 1991. Dados florísticos preliminares e elaboração da flórula vascular da Reserva Biológica do Ibicuí-Mirim, Santa Maria, Rio Grande do Sul, Brasil. Iheringia, 41: 141-153.

Orloci, L.; Pillar, V.D.; Anand, M. \& Behling, H. 2002. Some interesting characteristics of the vegetation process. Community Ecology 3: 125-146.

Pillar, V.D. 1999. The bootstrapped ordination reexamined. Journal of Vegetation Science 10: 895-902.
Pillar, V.D. 2008. MULTIV, sofware for multivariate exploratory analysis, randomization testing and bootstrap resampling (for Macintosh and Windows OS). Porto Alegre. http:// ecoqua.ecologia.ufrgs.br

Podani, J. 2000. Introduction to the Exploration of Multivariate Biological Data. Leiden, Backuys Publishers, 407 p.

Rambo, B. 1956. A fisionomia do Rio Grande do Sul. Selbach, Porto Alegre, $456 \mathrm{p}$.

Rambo, B. 1961. Migration routes of the South Brazilian rain forest. Pesquisas Série Botânica, 5:1-5.

Roubik, D.W. \& Moreno, J.E. 1991. Pollen and spores of Barro Colorado Island. Monographs in systematic botany from the Missouri Botanical Garden, Balboa, 269 p.

Salgado-Labouriau, M.L. 1973. Contribuição à Palinologia dos Cerrados. Editora Academia Brasileira de Ciências, Rio de Janeiro, $291 \mathrm{p}$.

Schmitz, P.I. 2005. O povoamento indígena do Sul do Brasil. In: Farias, D. S. E. de et al. (Org.) Maracajá: Pré-história e Arqueologia. Ed. Unisul, Tubarão, SC, 199 p.

Stockmarr, J. 1971. Tablets with spores used in absolute pollen analysis. Pollen et Spores, 13:615-621.

Weninger, B.; Jöris, O. \& Danzeglocke, U. 2004. Calpal-The Cologne radiocarbon CALibration and PALaeoclimate research package. http://www.calpal.de.

Received in June, 2008; accepted in August, 2008. 